\title{
A comparative study of recent neuroimaging techniques to show temporal region is the most common site in focal epilepsy irrespective of age
}

\author{
Jaishree Tapadia ${ }^{1, *}$, Rahul Mittal ${ }^{2}$ \\ ${ }^{\mathbf{1} A s s o c i a t e}$ Professor, Dept. of Physiology, SAIMS \& PG Institute, Indore, Madhya Pradesh, 2Assistant Professor, Dept. of \\ Physiology, Government Medical College, Gwalior, Madhya Pradesh, India
}

*Corresponding Author:

Email: jaishree_taparia@yahoo.in

\begin{abstract}
Epilepsy is a chronic disease characterized by recurrent seizures that may affect $2 \%$ of the population classification of seizures based solely on EEG and clinical findings can result in the misclassification of some patients, particularly those individuals with partial seizures that rapidly generalize. The information provided by imaging techniques can contribute to the proper classification of certain epileptic disorders in non-invasive localisation of epileptogenic foci. Neuroimaging has an important role in the investigation, localisation and treatment of patients with epilepsy. It is presently recognized that epilepsy is often associated with gross or subtle structural or metabolic lesions of the brain. Neuroimaging is mandatory in the work-up of epilepsy for localisation of the seizure focus for possible surgical cure. In this study it was concluded that that temporal lobe is most common site as epileptic lesion by different neuroimaging techniques.
\end{abstract}

Keywords: EEG, Electroencephalography, PET, Positron emission tomography, CT, Computed tomography, MRI, Magnetic resonance imaging, MRS, MR spectroscopy.

\section{Introduction}

Neuroimaging is mandatory in the work-up of epilepsy for localisation of the seizure focus for possible surgical cure. Epilepsy is a common disorder, with a prevalence of about $1 \%$ worldwide, and an estimated $7-11 \%$ of the population experiencing at least one seizure during their lifetime ${ }^{1}$ Neuroimaging is mandatory in the work-up of epilepsy for localisation of the seizure focus for possible surgical cure. Modern neuroimaging techniques have had a major impact on our understanding of epilepsy. ${ }^{1,2}$ They provide an exquisite degree of anatomical resolution and metabolic information about the epileptic lesion and contribute to the proper classification of certain epileptic disorders. Advances in technology to localize focal epileptogenic substrates, especially that of high-resolution structural imaging with magnetic resonance imaging (MRI) \& Positron Emission Tomography (PET) ${ }^{2}$ have substantially improved the success of surgical treatment. ${ }^{3}$ Modern neuroimaging techniques have directly and indirectly advanced our understanding of the basic pathophysiologic processes associated with the epilepsies. Yet despite this in up to $15 \%$ of patients with epilepsy, structural lesions can still remain undetected. According to one study the intention of epilepsy surgery is to achieve complete seizure control in medically intractable patients. This requires correct lateralization of the affected hemisphere with clear focus localization. Several studied suggest that although EEG remains the backbone for the evaluation of epilepsy, MR imaging currently is the method of choice for depicting gross structural lesions in the brain. ${ }^{4}$ ET and SPECT play a complementary role in the localization or lateralization of the epileptogenic focus and minimize the need for invasive EEG monitoring. ${ }^{5}$

\section{Materials and Methods}

Ethical Approval: This study approved from the Ethical \& Research Committee,

Study Design: Prospective observational study including 20 screened patients of epilepsy.

Consent for inclusion obtained from the patients in writing after explaining the whole procedure of the study and its utility before the procedure though the study was totally non-invasive. A detailed history recorded and detailed general and CNS examination done. After primary screening through EEG for onehour duration in awake state with hyperventilation and photic stimulation during interictal phase the patient would be subjected for neuroimaging of the brain in order to find epileptogenic focus, associated morphological changes in relation to EEG findings during interictal phases of epilepsy.

\section{Inclusion Criteria:}

1. Focal epilepsy patient attending neurology OPD \& referred by microphysicists (under treatment)

2. Age groups

3. Male and Female

4. Focal epilepsy

Exclusion Criteria:

1. Pregnancy.

2. Breast feeding. Mothers should interrupt breast feeding for $24 \mathrm{~h}$ if PET is indicated.

3. Lack of cooperation, or inability to cooperate, with the procedure.

\section{Equipment}

PET-CT Machine: PET-Scan Discovery STE-8(GE Medical systems) with an 8 slice CT-Scan. The dye being used in the machine is F-1 8 FDG (Fluoro-Deoxy Glucose) 
MRI Machine: Siemens 1.5 $\mathrm{T}$ with plain and spectroscopy study protocol data fused with help of cortex ID protocol on ADW 4.2 work station

\section{Method:}

1. Twenty (Ref), known patients (age: 5-65 yrs.) of either sex or suspected for symptomatically or clinically for epilepsy with different age group, sex and weight.

2. Parameter recorded

a) Physical

i. Age (years)

ii. Height $(\mathrm{cm})$

iii. Weight $(\mathrm{kg})$

b) Recording procedure
PET SCAN with all above criteria we have done CT scan, MRI, MR spectroscopy and PET scan.

\section{Statistical Analysis}

Data collected by prospective observational study entered in MS office Excel spread sheet. Statistical analysis done using NET based graph pad calculator. Correlation of findings between different neuroimaging modalities done using Chi Square test

Table 1: Localization of lesion in different hemisphere in different neuroimaging technique

\begin{tabular}{|l|c|c|c|c|}
\hline \multicolumn{1}{|c|}{ Parameter } & Temporal & Extra-temporal & Both & Total \\
\hline CT & $2(10 \%)$ & $1(5 \%)$ & $1(5 \%)$ & $4(20 \%)$ \\
\hline MRI & $3(15 \%)$ & $2(10 \%)$ & $2(10 \%)$ & $7(35 \%)$ \\
\hline MRS & $7(35 \%)$ & $2(10 \%)$ & $2(10 \%)$ & $11(55 \%)$ \\
\hline PET & $11(55 \%)$ & $2(10 \%)$ & $6(30 \%)$ & $19(95 \%)$ \\
\hline
\end{tabular}

Table indicating site of lesion by various neuroimaging techniques. It is clear that temporal site is the most recognized site for focal epilepsy independent of age.

Table 2: Localization rates between different neuroimaging technique $(\mathrm{N}=20)$

\begin{tabular}{|c|c|c|c|c|c|c|c|c|}
\hline \multicolumn{3}{|c|}{ MRI } & \multicolumn{3}{|c|}{ MRS } & \multicolumn{3}{|c|}{ PET } \\
\hline 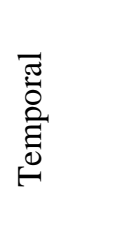 & 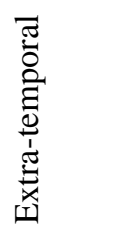 & 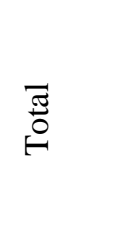 & 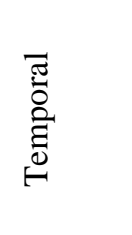 & 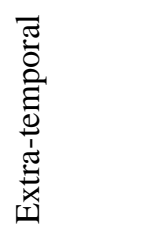 & 氶 & 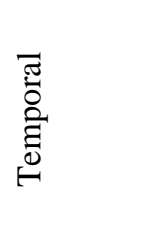 & 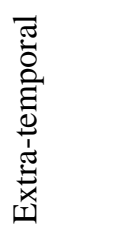 & $\begin{array}{l}\bar{\sigma} \\
\stackrel{\tilde{0}}{0}\end{array}$ \\
\hline $3(15 \%)$ & $2(10 \%)$ & $5(25 \%)$ & $7(35 \%)$ & $2(10 \%)$ & $9(45 \%)$ & $11(55 \%)$ & $2(10 \%)$ & $13(65 \%)$ \\
\hline
\end{tabular}

$\mathrm{P}=\leq 0.0023$ between MRI \&MRS

$\mathrm{P}=\leq 0.0001$ between MRI \&PET

$\mathrm{P}=0.4525$ between MRS\&PET

\section{Discussion}

Although intensive 24-hour video EEG monitoring is the "gold standard" for epileptic focus localization, multimodal approaches are coming to the fore because hemispheric lateralization is verified in case of concordance of different imaging modalities. ${ }^{6-8}$ This time is too long to image the relatively short ictal state. If PET is performed immediately after cessation of seizure activity, with immediate injection of the radiotracer during the seizure, the PET image shows poor uptake of the radiotracer throughout the brain. Among the interictal techniques, PET showed the highest sensitivity in patients with temporal lobe epilepsy (84\% versus $66 \%$ for interictal SPECT, $55 \%$ for qualitative MR imaging), in our studies we have found almost same result for temporal lobe epilepsy. PET showed the highest sensitivity in patients with temporal lobe epilepsy (55\% versus $40 \%$ for MRS, $15 \% \mathrm{MRI}$, and $10 \% \mathrm{CT})$. As in other studies, our investigation suggests that all imaging techniques had a lower sensitivity for extratemporal epilepsy than for temporal lobe epilepsy. Several studies have addressed the value of PET in TLE. ${ }^{136-138}$ Sensitivity for detecting relative temporal lobe hypometabolism with fluorodeoxyglucose-PET in TLE ranges from $80 \%$ to $90 \% .{ }^{9,10}$ our study is also favoring this result, in present study PET is $85 \%$ sensitive in diagnosing TLE. In the management of temporal lobe epilepsy, exact lateralization/localization of seizure focus with a noninvasive study is crucial, because surgical resection of the epileptogenic lesion results in good outcome (Engel class I or II) and a failure to lateralize the focus with noninvasive examination may lead to an invasive study or to additional surgery for placement of intracranial electrodes, which may have potential risks. ${ }^{11,12}$ Several FDG-PET studies in patients with temporal lobe epilepsy have shown a $60 \%$ to $90 \%$ prevalence of hypometabolism in the temporal lobe. ${ }^{13,14}$ 


\section{Conclusion}

Present studies indicate that all imaging methods shows that temporal region is most common for focal epilepsy irrespective of age.

The reasons why PET showed higher rates of correct lateralization in our study are uncertain. Several factors, including a different subject population, different imaging techniques, and a different standard of reference, might account for this discrepancy. Further studies are required for patients in whom lateralization of the seizure focus is inconclusive.

\section{References}

1. Ihle M, Feldwisch-Drentrup H, Teixeira CA, Witon A, Schelter B, Timmer J, Schulze-Bonhage A. EPILEPSIAE - A European epilepsy database. Comput Methods Programs Biomed 2010 Sep 20.

2. Pillai JJ, Hessler RB, Allison JD, Park YD, Lee MR, Lavin T. Advanced MR imaging of cortical dysplasia with or without neoplasm: a report of two cases. AJNR Am J Neuroradiol 2002;23:1686-1691.

3. Cendes F, Li LM, Watson C, et al. Is ictal recording mandatory in temporal lobe epilepsy? Not when the interictal electroencephalogram and hippocampal atrophy coincide. Arch Neurol 2000;57:497-500.

4. Stefan H, Pawlik G, Bocher-Schwarz HG, et al. Functional and morphological abnormalities in temporal lobe epilepsy: a comparison of interictal and ictal EEG, CT, MRI, SPECT and PET. J Neurol 1987;234:377-84.

5. Stefan H, Pawlik G, Bocher-Schwarz HG, et al. Functional and morphological abnormalities in temporal lobe epilepsy: a comparison of interictal and ictal EEG, CT, MRI, SPECT and PET. J Neurol 1987;234:377-84.

6. Soreni N, Noseworthy MD, Cormier T, et al. Intraindividual variability of striatal $1 \mathrm{H}-\mathrm{MRS}$ brain metabolite measurements at 3 T. Magn Reson Imaging 2006;24:187-94.

7. Riederer F, Bittsansky M, Schmidt C, et al. $1 \mathrm{H}$ magnetic resonance spectroscopy at $3 \mathrm{~T}$ in cryptogenic and mesial temporal lobe epilepsy. NMR Biomed 2006.

8. Hyung Jin Wona, Kee-Hyun Changa, et al Comparison of MR IMAGING with PET and ICTALSPECT in 118 patients with intractable epilepsy AJNR 1999 20: 593599.

9. Benbadis SR, So NK, Antar MA, et al. The value of PET scan (and MRI and Wada test) in patients with bitemporal epileptiform abnormalities. Arch Neurol 1995;52:106268.
10. Knowlton RC. The role of FDG-PET, ictal SPECT, and MEG in the epilepsy surgery evaluation. Epilepsy Behav 2006;8:91-101.

11. Van PW, Connelly A, Johnson CL, et al. The amygdala and intractable temporal lobe epilepsy: a quantitative magnetic resonance imaging study. Neurology 1996;47:1021-31.

12. Jack CR, Jr., Sharbrough FW, Cascino GD, et al. Magnetic resonance image-based hippocampal volumetry: correlation with outcome after temporal lobectomy. Ann Neurol 1992;31:138-46

13. Kim MA, Heo K, Choo MK, et al. Relationship between bilateral temporal hypometabolism and EEG findings for mesial temporal lobe epilepsy: analysis of 18F-FDG PET using SPM. Seizure 2006;15:56-63.

14. Gadian DG, Isaacs EB, Cross JH, et al. Lateralization of brain function in childhood revealed by magnetic resonance spectroscopy. Neurology 1996;46:974-77.

15. Spencer SS. The relative contributions of MRI, SPECT, and PET imaging in epilepsy. Epilepsia 1994;35:S72S89.

16. Duncan JS. Imaging and epilepsy. Brain 1997;120:339377.

17. Engel J Jr, Kuhl DE, Phelps ME, Crandall PH. Comparative localization of epileptic foci in partial epilepsy by PCT and EEG. Ann Neurol 1982;12:529-537.

18. Gailard WD, White S, Malow B, et al. FDG-PET in children and adolescents with partial seizures: role in epilepsy surgery evaluation. Epilepsy Res 1995;20:7-84.

19. Kuhl DE, Engel J Jr, Phelps ME, Selin C. Epileptic patterns of local cerebral metabolism and perfusion in humans determined by emission computed tomography of 18FDG and 13NH3. Ann Neurol 1980;8:348-360.

20. Ryvlin P, Cinotti L, Froment JC, et al. Metabolic patterns associated with non-specific magnetic resonance imaging abnormalities in temporal lobe epilepsy. Brain 1991;114:2363-2368.

21. Falconer MA. Mesial temporal (Anmon's horn) sclerosis as a common cause of epilepsy: aetiology, treatment and prevention. Lancet 1974;2:727-770.

22. Achten E, Santens P, Boon P, et al. Single-voxel proton MR spectroscopy and positron emission tomography for lateralization of refractory temporal lobe epilepsy. AJNR Am J Neuroradiol 1998;19:1-8. 